\title{
Multiple positive doubly periodic solutions for a singular semipositone telegraph equation with a parameter
}

Fanglei Wang ${ }^{1 *}$ and Yukun $\mathrm{An}^{2}$

\section{"Correspondence:}

wang-fanglei@hotmail.com

${ }^{1}$ College of Science, Hohai

University, Nanjing, 210098, P.R.

China

Full list of author information is

available at the end of the article

\begin{abstract}
In this paper, we study the multiplicity of positive doubly periodic solutions for a singular semipositone telegraph equation. The proof is based on a well-known fixed point theorem in a cone.

MSC: $34 \mathrm{~B} 15 ; 34 \mathrm{~B} 18$

Keywords: semipositone telegraph equation; doubly periodic solution; singular; cone; fixed point theorem
\end{abstract}

\section{Introduction}

Recently, the existence and multiplicity of positive periodic solutions for a scalar singular equation or singular systems have been studied by using some fixed point theorems; see [1-9]. In [10], the authors show that the method of lower and upper solutions is also one of common techniques to study the singular problem. In addition, the authors [11] use the continuation type existence principle to investigate the following singular periodic problem:

$$
\left(\left|u^{\prime}\right|^{p-2} u^{\prime}\right)^{\prime}+h(u) u^{\prime}=g(u)+c(t) .
$$

More recently, using a weak force condition, Wang [12] has built some existence results for the following periodic boundary value problem:

$$
\left\{\begin{array}{l}
u_{t t}-u_{x x}+c_{1} u_{t}+a_{11}(t, x) u+a_{12}(t, x) v=f_{1}(t, x, u, v)+\chi_{1}(t, x) \\
v_{t t}-v_{x x}+c_{2} v_{t}+a_{21}(t, x) u+a_{22}(t, x) v=f_{2}(t, x, u, v)+\chi_{2}(t, x)
\end{array}\right.
$$

The proof is based on Schauder's fixed point theorem. For other results concerning the existence and multiplicity of positive doubly periodic solutions for a single regular telegraph equation or regular telegraph system, see, for example, the papers [13-17] and the references therein. In these references, the nonlinearities are nonnegative.

On the other hand, the authors [18] study the semipositone telegraph system

$$
\left\{\begin{array}{l}
u_{t t}-u_{x x}+c_{1} u_{t}+a_{1}(t, x) u=b_{1}(t, x) f(t, x, u, v), \\
v_{t t}-v_{x x}+c_{2} v_{t}+a_{2}(t, x) v=b_{2}(t, x) g(t, x, u, v),
\end{array}\right.
$$

๑ 2013 Wang and An; licensee Springer. This is an Open Access article distributed under the terms of the Creative Commons Attribution License (http://creativecommons.org/licenses/by/2.0), which permits unrestricted use, distribution, and reproduction in any medium, provided the original work is properly cited. 
where the nonlinearities $f, g$ may change sign. In addition, there are many authors who have studied the semipositone equations; see $[19,20]$.

Inspired by the above references, we are concerned with the multiplicity of positive doubly periodic solutions for a general singular semipositone telegraph equation

$$
\left\{\begin{array}{l}
u_{t t}-u_{x x}+c u_{t}+a(t, x) u=\lambda f(t, x, u), \\
u(t+2 \pi, x)=u(t, x+2 \pi)=u(t, x),
\end{array}\right.
$$

where $c>0$ is a constant, $\lambda>0$ is a positive parameter, $a(t, x) \in C(R \times R, R), f(t, x, u)$ may change sign and is singular at $u=0$, namely,

$$
\lim _{u \rightarrow 0^{+}} f(t, x, u)=+\infty
$$

The main method used here is the following fixed-point theorem of a cone mapping.

Lemma 1.1 [21] Let $E$ be a Banach space, and $K \subset E$ be a cone in E. Assume $\Omega_{1}, \Omega_{2}$ are open subsets of $E$ with $0 \in \Omega_{1}, \bar{\Omega}_{1} \subset \Omega_{2}$, and let $T: K \cap\left(\bar{\Omega}_{2} \backslash \Omega_{1}\right) \rightarrow K$ be a completely continuous operator such that either

(i) $\|T u\| \leq\|u\|, u \in K \cap \partial \Omega_{1}$ and $\|T u\| \geq\|u\|, u \in K \cap \partial \Omega_{2}$; or

(ii) $\|T u\| \geq\|u\|, u \in K \cap \partial \Omega_{1}$ and $\|T u\| \leq\|u\|, u \in K \cap \partial \Omega_{2}$.

Then $T$ has a fixed point in $K \cap\left(\bar{\Omega}_{2} \backslash \Omega_{1}\right)$.

The paper is organized as follows. In Section 2, some preliminaries are given. In Section 3 , we give the main result.

\section{Preliminaries}

Let $\top^{2}$ be the torus defined as

$$
\top^{2}=(R / 2 \pi Z) \times(R / 2 \pi Z) .
$$

Doubly $2 \pi$-periodic functions will be identified to be functions defined on $T^{2}$. We use the notations

$$
L^{p}\left(\top^{2}\right), \quad C\left(\top^{2}\right), \quad C^{\alpha}\left(\top^{2}\right), \quad D\left(\top^{2}\right)=C^{\infty}\left(\top^{2}\right), \ldots
$$

to denote the spaces of doubly periodic functions with the indicated degree of regularity. The space $D^{\prime}\left(\top^{2}\right)$ denotes the space of distributions on $T^{2}$.

By a doubly periodic solution of Eq. (1) we mean that a $u \in L^{1}\left(T^{2}\right)$ satisfies Eq. (1) in the distribution sense, i.e.,

$$
\int_{\mathrm{T}_{2}} u\left(\varphi_{t t}-\varphi_{x x}-c \varphi_{t}+a(t, x) \varphi\right) d t d x=\lambda \int_{\mathrm{T}^{2}} f(t, x, u) \varphi d t d x .
$$

First, we consider the linear equation

$$
u_{t t}-u_{x x}+c u_{t}-\xi u=h(t, x), \quad \text { in } D^{\prime}\left(\top^{2}\right)
$$

where $c>0, \mu \in R$, and $h(t, x) \in L^{1}\left(T^{2}\right)$. 
Let $€_{\xi}$ be the differential operator

$$
£_{\xi} u=u_{t t}-u_{x x}+c u_{t}-\xi u
$$

acting on functions on $T^{2}$. Following the discussion in [14], we know that if $\xi<0, £_{\xi}$ has the resolvent $R_{\xi}$,

$$
R_{\xi}: L^{1}\left(T^{2}\right) \rightarrow C\left(T^{2}\right), \quad h_{i}(t, x) \mapsto u_{i}(t, x),
$$

where $u(t, x)$ is the unique solution of Eq. (2), and the restriction of $R_{\xi}$ on $L^{p}\left(T^{2}\right)(1<p<$ $\infty)$ or $C\left(\top^{2}\right)$ is compact. In particular, $R_{\xi}: C\left(T^{2}\right) \rightarrow C\left(T^{2}\right)$ is a completely continuous operator.

For $\xi=-c^{2} / 4$, the Green function $G(t, x)$ of the differential operator $\varepsilon_{\xi}$ is explicitly expressed; see Lemma 5.2 in [14]. From the definition of $G(t, x)$, we have

$$
\begin{aligned}
& \underline{G}:=\operatorname{ess} \inf G(t, x)=e^{-3 c \pi / 2} /\left(1-e^{-c \pi}\right)^{2}, \\
& \bar{G}:=\operatorname{ess} \sup G(t, x)=\left(1+e^{-c \pi}\right) / 2\left(1-e^{-c \pi}\right)^{2} .
\end{aligned}
$$

For convenience, we assume the following condition holds throughout this paper:

(H1) $a(t, x) \in C\left(T^{2}, R\right), 0 \leq a(t, x) \leq \frac{c^{2}}{4}$ on $T^{2}$, and $\int_{T^{2}} a(t, x) d t d x>0$.

Finally, if $-\xi$ is replaced by $a(t, x)$ in Eq. (2), the author [13] has proved the following unique existence and positive estimate result.

Lemma 2.1 Let $h(t, x) \in L^{1}\left(T^{2}\right)$. Then Eq. (2) has a unique solution $u(t, x)=P[h(t, x)], P$ : $L^{1}\left(\top^{2}\right) \rightarrow C\left(\top^{2}\right)$ is a linear bounded operator with the following properties:

(i) $P: C\left(\top^{2}\right) \rightarrow C\left(\top^{2}\right)$ is a completely continuous operator;

(ii) If $h(t, x)>0$, a.e $(t, x) \in T^{2}, P[h(t, x)]$ has the positive estimate

$$
\underline{G}\|h\|_{L^{1}} \leq P[h(t, x)] \leq \frac{\bar{G}}{\underline{G}\|a\|_{L^{1}}}\|h\|_{L^{1}} .
$$

\section{Main result}

Theorem 3.1 Assume (H1) holds. In addition, if $f(t, x, u)$ satisfies

(H2) $\lim _{u \rightarrow 0^{+}} f(t, x, u)=+\infty$, uniformly $(t, x) \in T^{2}$,

(H3) $f: \top^{2} \times(0,+\infty) \rightarrow(-\infty,+\infty)$ is continuous,

$(\mathrm{H} 4)$ there exists a nonnegative function $h(t, x) \in C\left(T^{2}\right)$ such that

$$
f(t, x, u)+h(t, x) \geq 0, \quad(t, x) \in T^{2}, u>0,
$$

(H5) $\int_{T^{2}} F_{\infty}(t, x) d t d x=+\infty$, where the limit function $F_{\infty}(t, x)=\liminf _{u \rightarrow+\infty} \frac{f(t, x, u)}{u}$, then Eq. (1) has at least two positive doubly periodic solutions for sufficiently small $\lambda$.

$C\left(\top^{2}\right)$ is a Banach space with the norm $\|u\|=\max _{(t, x) \in \top^{2}}|u(t, x)|$. Define a cone $K \subset$ $C\left(\top^{2}\right)$ by

$$
K=\left\{u \in C\left(\top^{2}\right): u \geq 0, u(t, x) \geq \delta\|u\|\right\},
$$


where $\delta=\frac{\underline{G}^{2}\|a\|_{L^{1}}}{\bar{G}} \in(0,1)$. Let $\partial K_{r}=\{u \in K:\|u\|=r\},[u]^{+}=\max \{u, 0\}$. By Lemma 2.1, it is easy to obtain the following lemmas.

Lemma 3.2 If $h(t, x) \in C\left(\top^{2}\right)$ is a nonnegative function, the linear boundary value problem

$$
\left\{\begin{array}{l}
u_{t t}-u_{x x}+c u_{t}+a(t, x) u=\lambda h(t, x), \\
u(t+2 \pi, x)=u(t, x+2 \pi)=u(t, x)
\end{array}\right.
$$

has a unique solution $\omega(t, x)$. The function $\omega(t, x)$ satisfies the estimates

$$
\lambda \underline{G}\|h\|_{L^{1}} \leq \omega(t, x)=\lambda P(h(t, x)) \leq \lambda \frac{\bar{G}}{\underline{G}\|a\|_{L^{1}}}\|h\|_{L^{1}} .
$$

Lemma 3.3 If the boundary value problem

$$
\left\{\begin{array}{l}
u_{t t}-u_{x x}+c u_{t}+a(t, x) u=\lambda\left[f\left(t, x,[u(t, x)-\omega(t, x)]^{+}\right)+h(t, x)\right] \\
u(t+2 \pi, x)=u(t, x+2 \pi)=u(t, x)
\end{array}\right.
$$

has a solution $\tilde{u}(t, x)$ with $\|\widetilde{u}\|>\lambda \frac{\bar{G}^{2}}{\underline{G}^{3}\|a\|_{L^{1}}^{2}}\|h\|_{L^{1}}$, then $u^{*}(t, x)=\widetilde{u}(t, x)-\omega(t, x)$ is a positive doubly periodic solution of Eq. (1).

Proof of Theorem 3.1 Step 1. Define the operator $T$ as follows:

$$
(T u)(t, x)=\lambda P\left[f\left(t, x,[u(t, x)-\omega(t, x)]^{+}\right)+h(t, x)\right] .
$$

We obtain the conclusion that $T\left(K \backslash\left\{u \in K:[u(t, x)-\omega(t, x)]^{+}=0\right\}\right) \subseteq K$, and $T: K \backslash\{u \in$ $\left.K:[u(t, x)-\omega(t, x)]^{+}=0\right\} \rightarrow K$ is completely continuous.

For any $u \in K \backslash\left\{u \in K:[u(t, x)-\omega(t, x)]^{+}=0\right\}$, then $[u(t, x)-\omega(t, x)]^{+}>0$, and $T$ is defined. On the other hand, for $u \in K \backslash\left\{u \in K:[u(t, x)-\omega(t, x)]^{+}=0\right\}$, the complete continuity is obvious by Lemma 2.1. And we can have

$$
\begin{aligned}
(T u)(t, x) & =\lambda P\left[f\left(t, x,[u(t, x)-\omega(t, x)]^{+}\right)+h(t, x)\right] \\
& \geq \lambda \underline{G}\left\|f\left(t, x,[u(t, x)-\omega(t, x)]^{+}\right)+h(t, x)\right\|_{L^{1}} \\
& \geq \underline{G} \frac{\underline{G}\|a\|_{L^{1}}}{\bar{G}}\|T(u)\| \\
& \geq \delta\|T u\| .
\end{aligned}
$$

Thus, $T(K \backslash\{u \in K: u(t, x) \leq \omega(t, x)\}) \subseteq K$.

Now we prove that the operator $T$ has one fixed point $\tilde{u} \in K$ and $\|\tilde{u}\|>\lambda \frac{\bar{G}^{2}}{\underline{G}^{3}\|a\|_{L^{1}}^{2}}\|h\|_{L^{1}}$ for all sufficiently small $\lambda$.

Since $\int_{\top^{2}} F_{\infty}(t, x) d t d x=+\infty$, there exists $r_{1} \geq 2$ such that

$$
\int_{\top^{2}} \frac{f(t, x, u)}{u} d t d x \geq \frac{1}{\delta}, \quad u \geq \delta r_{1} .
$$


Furthermore, we have $\int_{T^{2}} f\left(t, x, \delta r_{1}\right) d t d x \geq r_{1} \geq 2$. It follows that

$$
\begin{gathered}
\int_{\top^{2}}\left[\max \left\{f(t, x, u): \frac{\delta}{2} r_{1} \leq u \leq r_{1}\right\}+h(t, x)\right] d t d x \\
\geq \int_{\top^{2}} f\left(t, x, \delta r_{1}\right) d t d x \geq r_{1} \geq 2 .
\end{gathered}
$$

Let $\Phi(t, x)=\max \left\{f(t, x, u): \frac{\delta}{2} r_{1} \leq u \leq r_{1}\right\}+h(t, x)$. Then $\Phi \in L^{1}\left(\top^{2}\right)$ and $\int_{\top^{2}} \Phi(t, x) d t d x>$ 0 . Set

$$
\lambda^{*}=\min \left\{\frac{\delta^{2}}{2 \underline{G}\|h\|_{L^{1}}}, \frac{2 \underline{G}\|a\|_{L^{1}}}{\bar{G}\|\Phi\|_{L^{1}}}\right\} .
$$

For any $u \in \partial K_{r_{1}}$ and $0<\lambda<\lambda^{*}$, we can verify that

$$
\begin{aligned}
u(t, x)-\omega(t, x) & \geq \delta\|u\|-\omega(t, x) \\
& =\delta r_{1}-\omega(t, x) \\
& \geq \delta r_{1}-\lambda \frac{\bar{G}}{\underline{G}\|a\|_{L^{1}}}\|h\|_{L^{1}} \\
& \geq \delta r_{1}-\frac{\delta r_{1}}{2} \\
& =\frac{\delta r_{1}}{2} .
\end{aligned}
$$

Then we have

$$
\begin{aligned}
\|T u\| & =\lambda\left\|P\left[f\left(t, x,[u(t, x)-\omega(t, x)]^{+}\right)+h(t, x)\right]\right\| \\
& \leq \lambda \frac{\bar{G}}{\underline{G}\|a\|_{L^{1}}}\left\|f\left(t, x,[u(t, x)-\omega(t, x)]^{+}\right)+h(t, x)\right\|_{L^{1}} \\
& \leq \lambda \frac{\bar{G}}{\underline{G}\|a\|_{L^{1}}}\|\Phi(t, x)\|_{L^{1}} \\
& <2 \leq r_{1}=\|u\| .
\end{aligned}
$$

On the other hand,

$$
\liminf _{u \rightarrow+\infty} \frac{f(t, x, u-\omega(t, x))}{u}=\liminf _{u \rightarrow+\infty} \frac{f(t, x, u)}{u}=F_{\infty}(t, x) .
$$

By the Fatou lemma, one has

$$
\begin{gathered}
\liminf _{u \rightarrow+\infty} \int_{T^{2}} \frac{f(t, x, u-\omega(t, x))+h(t, x)}{u} d t d x \\
\geq \int_{\top^{2}} \liminf _{u \rightarrow+\infty} \frac{f(t, x, u)+h(t, x)}{u} d t d x \\
=\int_{\top^{2}} F_{\infty}(t, x) d t d x=+\infty .
\end{gathered}
$$


Hence, there exists a positive number $r_{2}>\delta r_{2}>r_{1}$ such that

$$
\int_{\top^{2}} \frac{f(t, x, u-\omega(t, x))+h(t, x)}{u} d t d x \geq \lambda^{-1} \delta^{-1} \underline{G}^{-1}\left(4 \pi^{2}\right)^{-1}, \quad u \geq \delta r_{2} .
$$

Hence, we have

$$
\int_{T^{2}} f(t, x, u-\omega(t, x))+h(t, x) d t d x \geq \lambda^{-1} \underline{G}^{-1}\left(4 \pi^{2}\right)^{-1} r_{2}, \quad u \geq \delta r_{2} .
$$

For any $u \in \partial K_{r_{2}}$, we have $\delta r_{2}=\delta\|u\| \leq u(t, x) \leq\|u\|=r_{2}$. On the other hand, since $0<\lambda<\lambda^{*}$, we can get

$$
\begin{aligned}
u(t, x)-\omega(t, x) & \geq \delta r_{2}-\omega(t, x) \\
& \geq \delta \frac{r_{2}}{\delta}-\lambda \frac{\bar{G}}{\underline{G}\|a\|_{L^{1}}} \\
& \geq \delta r_{2}-\delta \\
& >0 .
\end{aligned}
$$

From above, we can have

$$
\begin{aligned}
\|T u\| & \geq \lambda P\left[f\left(t, x,[u(t, x)-\omega(t, x)]^{+}\right)+h(t, x)\right] \\
& \geq \lambda \underline{G}\left\|f\left(t, x,[u(t, x)-\omega(t, x)]^{+}\right)+h(t, x)\right\|_{L^{1}} \\
& \geq \lambda \underline{G} 4 \pi^{2} \lambda^{-1} \underline{G}^{-1}\left(4 \pi^{2}\right)^{-1} r_{2} \\
& =r_{2} .
\end{aligned}
$$

Therefore, by Lemma 1.1, the operator $T$ has a fixed point $\widetilde{u}(t, x) \in K$ and

$$
\begin{aligned}
& r_{2} \geq\|\tilde{u}\| \geq r_{1}, \\
& \tilde{u}(t, x)-\omega(t, x) \geq \delta r_{1}-\lambda \frac{\bar{G}}{\underline{G}\|a\|_{L^{1}}}\|h\|_{L^{1}} \geq \delta r_{1}-\frac{\bar{G}}{\underline{G}\|a\|_{L^{1}}}\|h\|_{L^{1}} \frac{\delta^{2}}{\underline{G}\|h\|_{L^{1}}} \geq \delta .
\end{aligned}
$$

So, Eq. (1) has a positive solution $\widehat{u}(t, x)=\widetilde{u}(t, x)-\omega(t, x) \geq \delta$.

Step 2. By conditions (H2) and (H3), it is clear to obtain that

$$
u_{0}=\inf \left\{u \in K: f(t, x, u) \leq 0,(t, x) \in \top^{2}\right\}>0 .
$$

Let $r_{4}=\min \left\{\frac{\delta}{2}, \frac{\delta\left\|u_{0}\right\|}{2}\right\}$. For any $u \in\left(0, r_{4}\right]$, we have $f(t, x, u)>0$. Then define the operator $A$ as follows:

$$
(A u)(t, x)=\lambda \widehat{P}[f(t, x, u(t, x))] .
$$

It is easy to prove that $A\left(K \cap\left\{u \in C\left(\top^{2}\right): 0<\|u\|<r_{4}\right\}\right) \subseteq K$, and $A: K \cap\left\{u \in C\left(\top^{2}\right): 0<\right.$ $\left.\|u\|<r_{4}\right\} \rightarrow K$ is completely continuous.

And for any $\rho>0$, define

$$
M(\rho)=\max \left\{f(t, x, u): u \in R^{+}, \delta \rho \leq u \leq \rho,(t, x) \in \top^{2}\right\}>0 .
$$


Furthermore, for any $u \in \partial K_{r_{4}}$, we have

$$
\begin{aligned}
\|A u\| & =\lambda\|\widehat{P}[f(t, x, u(t, x))]\| \\
& \leq \lambda \frac{\bar{G}}{\underline{G}\|a\|_{L^{1}}}\|f(t, x, u(t, x))\|_{L^{1}} \\
& \leq \lambda \frac{\bar{G}}{\underline{G}\|a\|_{L^{1}}} M\left(r_{4}\right) 4 \pi^{2} .
\end{aligned}
$$

Thus, from the above inequality, there exists $\bar{\lambda}$ such that

$$
\|A u\|<\|u\|, \quad \text { for } u \in \partial K_{r_{4}}, 0<\lambda<\bar{\lambda} .
$$

Since $\lim _{u \rightarrow 0^{+}} f(t, x, u)=\infty$, then there is $0<r_{3}<\frac{r_{4}}{2}$ such that

$$
f(t, x, u) \geq \mu u, \quad \text { for } u \in R^{+} \text {with } 0<u \leq r_{3},
$$

where $\mu$ satisfies $\lambda \underline{G} \mu \delta>1$. For any $u \in \partial K_{r_{3}}$, then we have

$$
f(t, x, u) \geq \mu u(t, x), \quad \text { for }(t, x) \in \top^{2} .
$$

By Lemma 2.1, it is clear to obtain that

$$
\begin{aligned}
\|A u\| & =\lambda\|\widehat{P}[f(t, x, u(t, x))]\| \\
& \geq \lambda \underline{G}\|f(t, x, u(t, x))\|_{L^{1}} \\
& \geq \lambda \underline{G} \mu \delta r_{3} \\
& >r_{3}=\|u\| .
\end{aligned}
$$

Therefore, by Lemma 1.1, $A$ has a fixed point in $\bar{u}(t, x) \in K$ and $\|\bar{u}\| \leq r_{4} \leq \frac{\delta}{2}$, which is another positive periodic solution of Eq. (1).

Finally, from Step 1 and Step 2, Eq. (1) has two positive doubly periodic solutions $\widehat{u}(t, x)$ and $\bar{u}(t, x)$ for sufficiently small $\lambda$.

Example Consider the following problem:

$$
\left\{\begin{array}{l}
u_{t t}-u_{x x}+2 u_{t}+\sin ^{2}(t+x) u=\lambda\left[\frac{1}{u}+\min \left\{u^{2}, \frac{u}{\left|1-\frac{t}{\pi}\right|\left|1-\frac{x}{\pi}\right|}\right\}-10\right] \\
u(t+2 \pi, x)=u(t, x+2 \pi)=u(t, x)
\end{array}\right.
$$

It is clear that $f(t, x, u)$ satisfies the conditions $(\mathrm{H} 1)-(\mathrm{H} 5)$. 


\section{Author details}

${ }^{1}$ College of Science, Hohai University, Nanjing, 210098, P.R. China. ${ }^{2}$ Department of Mathematics, Nanjing University of Aeronautics and Astronautics, Nanjing, 210016, P.R. China.

\section{Acknowledgements}

The authors would like to thank the referees for valuable comments and suggestions for improving this paper.

Received: 26 July 2012 Accepted: 29 December 2012 Published: 16 January 2013

\section{References}

1. Chu, J, Torres, PJ, Zhang, M: Periodic solutions of second order non-autonomous singular dynamical systems. J. Differ Equ. 239, 196-212 (2007)

2. Chu, J, Fan, N, Torres, PJ: Periodic solutions for second order singular damped differential equations. J. Math. Anal. Appl. 388, 665-675 (2012)

3. Chu, J, Zhang, Z: Periodic solutions of second order superlinear singular dynamical systems. Acta Appl. Math. 111 179-187 (2010)

4. Chu, J, Li, M: Positive periodic solutions of Hill's equations with singular nonlinear perturbations. Nonlinear Anal. 69 , 276-286 (2008)

5. Chu, J, Torres, PJ: Applications of Schauder's fixed point theorem to singular differential equations. Bull. Lond. Math. Soc. 39, 653-660 (2007)

6. Jiang, D, Chu, J, Zhang, M: Multiplicity of positive periodic solutions to superlinear repulsive singular equations. J. Differ. Equ. 211, 282-302 (2005)

7. Torres, PJ: Existence of one-signed periodic solutions of some second-order differential equations via a Krasnoselski fixed point theorem. J. Differ. Equ. 190, 643-662 (2003)

8. Torres, PJ: Weak singularities may help periodic solutions to exist. J. Differ. Equ. 232, 277-284 (2007)

9. Wang, H: Positive periodic solutions of singular systems with a parameter. J. Differ. Equ. 249, 2986-3002 (2010)

10. DeCoster, C, Habets, P: Upper and lower solutions in the theory of ODE boundary value problems: classical and recent results. In: Zanolin, F (ed.) Nonlinear Analysis and Boundary Value Problems for Ordinary Differential Equations CISM-ICMS, vol. 371, pp. 1-78. Springer, New York (1996)

11. Jebelean, $P$, Mawhin, J: Periodic solutions of forced dissipative $p$-Liénard equations with singularities. Vietnam J. Math 32, 97-103 (2004)

12. Wang, F: Doubly periodic solutions of a coupled nonlinear telegraph system with weak singularities. Nonlinear Anal., Real World Appl. 12, 254-261 (2011)

13. Li, Y: Positive doubly periodic solutions of nonlinear telegraph equations. Nonlinear Anal. 55, 245-254 (2003)

14. Ortega, R, Robles-Perez, AM: A maximum principle for periodic solutions of the telegraph equations. J. Math. Anal. Appl. 221, 625-651 (1998)

15. Wang, F, An, Y: Nonnegative doubly periodic solutions for nonlinear telegraph system. J. Math. Anal. Appl. 338, 91-100 (2008)

16. Wang, F, An, Y: Existence and multiplicity results of positive doubly periodic solutions for nonlinear telegraph system. J. Math. Anal. Appl. 349, 30-42 (2009)

17. Wang, F, An, Y: Nonnegative doubly periodic solutions for nonlinear telegraph system with twin-parameters. Appl. Math. Comput. 214, 310-317 (2009)

18. Wang, F, An, Y: On positive solutions of nonlinear telegraph semipositone system. Dyn. Contin. Discrete Impuls. Syst., Ser. A Math. Anal. 16, 209-219 (2009)

19. Xu, X: Positive solutions for singular semi-positone three-point systems. Nonlinear Anal. 66, 791-805 (2007)

20. Yao, Q: An existence theorem of a positive solution to a semipositone Sturm-Liouville boundary value problem. Appl. Math. Lett. 23, 1401-1406 (2010)

21. Guo, D, Lakshmikantham, V: Nonlinear Problems in Abstract Cones. Academic Press, New York (1988)

doi:10.1186/1687-2770-2013-7

Cite this article as: Wang and An: Multiple positive doubly periodic solutions for a singular semipositone telegraph equation with a parameter. Boundary Value Problems 2013 2013:7.

\section{Submit your manuscript to a SpringerOpen ${ }^{\odot}$ journal and benefit from:}

- Convenient online submission

- Rigorous peer review

Immediate publication on acceptance

Open access: articles freely available online

- High visibility within the field

- Retaining the copyright to your article 